\title{
Rare and undiagnosed liver diseases: challenges and opportunities
}

\section{The complex landscape of rare diseases}

According to the statement of the European Commission of Public Health, rare diseases (RDs) are a group of 'life-threatening or chronically debilitating diseases which are of such low prevalence that special combined efforts are needed to address them' (1). Epidemiological criteria are essential for their definition. In Europe, a disease whose prevalence is lower than one in 2,000 people is considered a RD, while in the USA prevalence of a RD is less than one in 1,500 people, derived from the definition of a disease affecting fewer than 200,000 Americans. The difference in the prevalence thresholds between Europe and the USA reflects the wide variability among individual jurisdictions dependent on the current estimates of the population size in the different countries (2). At present, about 7,000 different diseases are recognized as 'rare', and altogether, affect about 400 million people worldwide (3). In a considerable percentage of patients (nearly $30 \%$ ), the diseases prolong into the adult age or manifest in the adult age. Thus, this is a field of medicine that concerns both pediatrician and adult physicians and spans across all medical and surgical specialties.

Most RDs (about $80 \%$ ) are caused by monogenic defects. However, a perturbed homeostatic response of the cell may activate other disease mechanisms, including autoimmunity and autoinflammation, susceptibility to infectious agents and cancer. In addition, the correlation between genotype and phenotype is often quite variable. In fact, in a number of RDs, affected genes control fundamental development processes resulting in a multifaceted clinical phenotype and multiorgan involvement.

According to the Orphanet database, about $25 \%$ of rare patients die before 5 years, whereas an additional $37 \%$ have a reduced life expectancy, variably dependent on the severity of the RD (4). Too often, the diagnosis represents a big challenge. Because of the limited awareness of these conditions and the scarcity of centers with adequate clinical experience and laboratory skills, the diagnosis is difficult to achieve and is thus often delayed. Notably, the average time for a patient with a rare disease to receive an accurate diagnosis is nearly 5 years (3). Unfortunately, despite repeated cost- and time-consuming efforts, several (6\%) remain undiagnosed (5).

Even when a diagnosis is reached, often the treatment options are limited. In spite of the recent progresses, it is estimated that $95 \%$ of RDs still lack an FDA approved treatment. It may take 10-12 years for a new drug to be approved for the cure of rare disease patients (6). This long avenue is also partly dependent on the difficulties in conducting clinical trials in rare diseases (small cohorts of widely dispersed patients in both pediatric and adult age, lack of validated biomarkers and qualified endpoints). Therefore, RDs are also often referred to as 'orphan' to highlight the concept that effective treatments are lacking as not enough research and drug development efforts are devoted to that problem. It is worth noting that only one in two RDs has a foundation or research support group, thereby limiting the access to grant opportunities (3). The majority of research funding is assigned to the most frequent diseases or as a reaction to particular events. It should be noted however, that funding research on RDs would also benefit the more prevalent diseases, as a result of the novel cellular functions and targets discovered.

\section{Rare diseases of the liver}

Liver involvement is a relevant feature in many RDs, where it may be either a major cause of morbidity and mortality, or a collateral manifestation in the context of a systemic condition with multiple organ damage and with limited impact on the natural history of the disease. Furthermore, the epidemiological criteria to define the 'rarity' of a liver disease is dependent on their geolocalization. This is a well-recognized concept when dealing with RDs of infectious origin, since etiologies that are common in Asian countries, as schistosomiasis, are rare in Europe. However, this is also true for diseases like primary sclerosing cholangitis (PSC), which, within Europe, is rare in the Mediterranean area, but not in the Scandinavian countries. Hemocromatosis is another example of RD with unique geo-epidemiological features, related to a 'Celtic mutation'. Hemochromatosis however may be caused by other quite rare mutations, as nicely discussed in the paper from Piperno et al. (7). 
On a pathogenetic ground, rare liver diseases are highly heterogeneous, and are driven by multiple and scarcely understood mechanisms. Thus, an attempt at classification is difficult. According to the proposal of the European Reference Network (ERN) on rare liver diseases, these can be categorized as autoimmune, infectious (viral, bacterial and parasitic), genetic/ hereditary (with distinct metabolic, cholestatic and structural phenotypes), vascular, neoplastic, and others of unknown etiology, involving multiple factors, as biliary atresia (8). Although these diseases are difficult to study because of the limited access to large series and biological samples, they have a remarkable translational value. In fact, as hinted below, rare liver diseases may provide us with unique models to unravel liver pathophysiological responses, like the liver repair mechanisms responsible for disease progression. In monogenic conditions, perturbation of homeostatic mechanisms by a single faulty gene can be probed, taking advantage of well-established and well-reproducible experimental conditions, as discussed by Fiorotto et al. (9). Thus, these diseases may be regarded as a 'pathophysiological roadmap' to decipher 'core' mechanisms that can be relevant also for more common chronic liver diseases (10).

Starting from these considerations, in this focused series we discuss a number of rare liver diseases. We did not aim to be exhaustive, but rather we selected diseases and topics according to a fourfold purpose. First, to highlight disorders that are paradigmatic for novel concepts in translational hepatology. Second, to outline innovative diagnostic approaches (liver histology advances, omics and cell-based technologies) that may enable us to recognize rare liver diseases more accurately and faster. Third, to underline the importance of the undiagnosed disease program in this setting. Fourth, to discuss treatment perspectives (liver transplantation, genome editing technologies) that may support rare disease management now and in the near future.

\section{Lessons from rare liver diseases}

\section{Genetic metabolic liver diseases}

Inherited iron overload disorders have been intensely investigated, with identification of many clinical forms, characterized by systemic involvement. They are determined by gene defects involved in hepcidin regulation, cellular iron uptake, handling and export, and iron transport and storage. Tissue iron overload derives from a complex interplay between intestinal iron absorption and macrophage iron release, where the degree of hepcidin dysfunction represents the main determinant of the clinical severity. This topic is elegantly reviewed by Piperno et al. (7), which also highlight the sequential strategy of integrating clinical, imaging, biochemical and genetic findings, necessary to reach the diagnosis, and the treatment based on blood removal and iron chelation.

Wilson disease is another well-recognized example of metabolic liver disease of genetic origin resulting in excess storage of a trace element, copper, in different tissues particularly in liver, brain and corneas. Moini et al. (11) thoroughly discuss the recent advances in the diagnostic work-up of Wilson disease, highlighting new methodologies for newborn screening, adult diagnosis and early detection of neurologic involvement, as well as for disease monitoring through assessment of non-ceruloplasmin bound copper and exchangeable copper in the circulation. The authors also illustrate therapeutic approaches including novel chelators and genetic manipulation for ATP7B introduction into hepatocytes that are currently in development.

Given their complexity, acute hepatic porphyrias have been often viewed as one of the trickiest and most difficult to recognize liver diseases. The acute hepatic porphyrias comprise four genetic conditions with low penetrance characterized by recurrent attacks of neurovisceral manifestations. They are caused by defects in heme biosynthesis resulting in increased levels of porphyrin precursors, among which delta-aminolevulinic acid (ALA). In their paper, Wang et al. (12) provide a comprehensive overview of these conditions, underlining the concept that excess hepatic ALA production mediates neurotoxicity in acute attacks as a paradigm of the close functional interactions between liver and nerve cells. Although biochemical tools are necessary to reach the diagnosis, genetic testing is the gold standard for confirmation. A mainstay in management of acute attacks is identifying and eliminating known triggers, as exemplified in many chronic liver diseases.

Accumulation of intermediate compounds with high reactivity may trigger a variety of cellular responses aimed at reducing its burden. This mechanism is well exemplified in $\alpha 1$-antitrypsin (A1AT) deficiency. As clearly addressed by Teckman et al. (13), accumulation of misfolded mutant $Z$ proteins activates in hepatocytes ubiquitin-dependent and ubiquitin-independent proteasomal pathways, endoplasmic reticulum-associated degradation and autophagy, which are responsible for liver injury. 
Importantly, not all subjects with homozygous $\mathrm{ZZ}$ genotype develop liver disease, in line with the concept that genetic factors cooperate with environmental modifiers of the intracellular injury cascade and fibrotic response to generate the disease phenotype. This disease is still orphan and improved understanding of liver injury mechanisms is needed to identify novel therapeutic approaches aimed at enhancing proteolysis.

\section{Genetic cholestatic liver diseases}

Genetic defects affecting both hepatocytes and cholangiocytes are an important cause of cholestatic diseases. There are numerous forms of genetic cholestatic diseases and they can target the hepatocyte or the cholangiocyte. Sometimes, as in ABCB4 deficiency, the defect affects a hepatocellular transporter, but the effect is a cholangitis. When the target is on the intrahepatic bile ducts, it may result in both bile duct paucity (ductopenia) and overgrowth (microhamartomas and cysts). In children, bile duct paucity represents a relevant diagnostic finding, requiring a broad differential diagnosis with the need of supplementary testing and characterization. Among ductopenic disorders, Alagille syndrome has been extensively studied in the last 30 years, paving the way towards a better understanding of biliary development and regeneration, and of liver repair in general. In this context, Gilbert et al. (14) have unfolded the fascinating 'Notch signaling story' and its strong impact on multiple genetic, pathology, diagnostic and therapeutic aspects of modern hepatology. Taking advantage of numerous in-vitro and in-vivo disease models, recent studies in Alagille syndrome hold promise for future generation of gene-based and proteinbased therapies, possibly applied to other chronic liver disorders from more common etiologies.

Proteins expressed in the primary cilia of cholangiocytes represent another set of morphogens regulating biliary development. Among them, fibrocystin is encoded by the PKHD1 gene, whose defect leads to a spectrum of disorders mainly affecting the kidney and the liver, which encompass congenital hepatic fibrosis and Caroli disease/syndrome. Interestingly, biliary dysgenesis develops in conjunction with peribiliary fibrosis, related to a pro-inflammatory phenotype that cholangiocytes activate when fibrocystin function is impaired. Since biliary fibrosis is a main determinant of portal hypertension, fibrocystic liver diseases provide a useful model to target mechanisms of disease progression in hepatic conditions. These concepts are reviewed by our group (15), emphasizing the notion that while current treatments of fibrocystic liver diseases are still limited to surgery, interventional radiology and liver transplantation, translational studies have identified a number of promising molecular targets.

\section{Immune-mediated and inflammatory liver diseases}

Propensity of liver epithelial cells to immune-mediated attacks is largely recognized, but interestingly, response to immunosuppression is unsuccessful when the primary site of this type of injury is the bile ducts. Liver autoimmunity comprises also peculiar disorders exclusively affecting children. Giant cell hepatitis associated with autoimmune hemolytic anemia is a form of severe acute liver injury, most common in early childhood, and histologically characterized by a giant cell transformation of hepatocytes. Nastasio et al. have drawn our attention on this rare condition, which is underdiagnosed also due to the lack of specific laboratory tests (16). Unique among liver diseases, recent evidence suggest that liver injury is promoted by a dysregulated humoral immune response sustained by a systemic B-cell autoimmunity directed against hepatocytes and erythrocytes. Compared with juvenile autoimmune hepatitis, response to immunosuppression is less favorable in giant cell hepatitis, with a high mortality and recurrence rate following liver transplantation.

Among inflammatory cholangiopathies, PSC is considered one of 'the remaining great challenges in hepatology' (17); despite the efforts made in the last two decades, the pathophysiological understanding of this enigmatic disease has remained scarce, and therefore, curative treatments are still lacking apart from liver transplantation. Silveira et al. (18) have carefully examined the several unexplored issues by which PSC continues to be shrouded in mystery. Of particular interest is indeed their discussion of the perspective treatment options.

\section{Rare liver cancers}

Among hepatic malignant tumors, which are classically considered highly lethal because of their marked aggressiveness, 
hepatoblastoma represents a virtuous model, because of the significant improvements in clinical management obtained, thanks to accurate risk stratification studies and development of precision medicine. Calvisi and Solinas have finely summarized the new insights on the biology of hepatoblastoma, by addressing the driver genes with major pathogenetic relevance (CTNNB1, NFE2L2, and TERT promoter, among others) and the relative pathways amenable of therapeutic intervention, including the emerging role of chimeric-antigen receptor therapy targeting glypican-3 and EpCam (19). In hepatoblastoma, some in-vivo models suitable to test new treatment strategies have been recently generated, and hopefully gaps in knowledge will be bridged further.

\section{Innovative diagnostic approaches of rare liver disease}

Among the challenges faced by patients with rare diseases, the difficulty in reaching an accurate diagnosis in reasonable time is at the top of the list. In rare liver diseases, the approaches usually taken for the diagnosis of more common liver diseases are inadequate. In fact, in spite of the success of non-invasive methods to evaluate liver-related parameters, liver biopsy remains a pillar in the intricate diagnostic work-up of rare liver diseases. Indeed, liver tissue sampling will play an increasingly important role, fostered by the emergence of new technologies for morphologic assessment, tissue content analysis of metabolites, proteins, gene expression and methylation. These concepts are elegantly outlined by Jain et al. (20), which describe the range of innovative approaches enabled by the use of different light sources and/or microscopes, such as the clearing histology with multiphoton microscopy (CHiMP), and the matrix-assisted laser desorption imaging (MALDI) mass spectrometry. These new methodologies will also support more effective translational research in rare liver diseases, enabling the discovery of diagnostic and prognostic biomarkers, as well as the dissection of new molecular mechanisms, and their relative putative targets. Furthermore, data obtained with histological analysis can be integrated into machine learning algorithms that, with the aid of artificial intelligence, will likely lead to more precise assessment of phenotypes, diagnoses and prognoses. This approach can be enforced further by molecular and clinical data acquired with the new omics technologies (genomics, proteomics, metabolomics), and then processed with bio-computational analysis.

These methods are extensively reviewed by Oliveira and Fiorotto (9) that focus our attention on the new advances in disease modeling, using cell-based technologies, including induced pluripotent stem cells (iPSC), and tissue organoids. These methods are particularly relevant for rare diseases, as they provide an effective tool to generate disease-specific biological insights, including molecular target identification, as well as individual profiling, and drug testing. These are prerequisite to pave the way to precision medicine and likely, to personalized treatments. Remarkably, both liver iPSC and organoids have been used for modeling monogenic rare liver diseases, such as Alagille syndrome, polycystic liver disease, Wilson disease and A1AT deficiency, and cystic fibrosis cholangiopathy.

Closely interwoven with the efforts to reach a correct diagnosis is the problem of conditions that elude standard diagnostic work-up and remain undiagnosed. Indeed, this can happen in a relevant proportion (nearly 20\%) of patients with chronic liver biochemistry abnormalities. Gao et al. (21) drive us through an exciting journey into the problem of the undiagnosed hepatic diseases. This definition includes patients that remain without a diagnosis in spite of costly and time-consuming efforts. Most of the times, these patients reach a diagnosis when referred to a Medical Center with the appropriate skills and tools, but sometimes the patients are actually affected by a novel disorder not previously described. In this multifaceted landscape, the authors propose a framework based on the categorization of undiagnosed liver diseases into four clinical groups: (I) the hidden liver disease, which consists of underdiagnoses or misdiagnosis of well-characterized hepatic disease; (II) the disguised liver disease, which includes hepatic manifestations of broader well-characterized diseases better known for extrahepatic presentation (i.e., cystic fibrosis); (III) the known liver disease of unknown cause, such as well-characterized liver phenotypes with unknown etiology (i.e., biliary atresia, PSC), and (IV) and the unknown liver diseases, encompassing novel liver diseases with uncharacterized phenotypic features, natural history and unknown cause. We believe that group (III) and (IV) are conditions that suit perfectly to the novel approaches to liver disease diagnosis and modeling, as discussed in (9).

\section{Treatment of rare liver disease}

As mentioned above, many liver diseases are orphan in terms of curative treatments; in some of them, liver transplantation (LT) stands as the only curative option. Of note, less than $1 \%$ of LT is performed for rare diseases, among which the main 
indications are non-hepatocellular malignancy, vascular disorders, and metabolic and congenital liver disorders. Being aware of these nonstandard indications to LT and providing an early referral for transplant may improve the overall survival and the quality of life in these patients. Moreover, close collaborations between transplant centers with molecular hepatology laboratories are strongly encouraged to make available valuable biological materials for translational studies devoted to these conditions. Finotti et al. (22) cover this important topic. Furthermore, the authors discuss the topic of 'rare' indications to LT from non-rare diseases, such as colorectal liver metastases or cholangiocarcinoma. Due to the contraction in conventional indications to LT (such as HCV-related cirrhosis), it is expected that in the near future these conditions may become more common indications, by expanding criteria for marginal donors, and by increasing the use of living donor LTs.

Rare liver diseases are often caused by production of defective or non-functional proteins crucial for fundamental activities of liver epithelial cells, such as differentiation, secretion, proliferation. Thus, gene therapy has been traditionally considered as an attractive approach to restore adequate protein levels through delivery of transgenes. Trevisan $e t$ al. give an excellent overview of this topic, by addressing many different genome-editing platforms, which are shifting the field from overexpression of defective gene products to direct correction of altered genomic DNA sequences (23). With the very recent introduction of base editors, a further step toward single nucleotide-precise correction has definitely been made. Although such technology is still in its early phases, hopefully in the future, it will become a game-changer for the current generation of molecular hepatologists and their patients.

\section{Conclusions}

While rare as a single entity, RDs are frequent as a group. The approach and tools necessary for their diagnosis, understanding, and target identification are complex, but do not differ much between different RDs. In editing this focused series on the novel advances in rare and undiagnosed liver diseases, we hoped to highlight, using specific examples, the burden of these diseases, their complexity, the unmet needs for the patients and their families, as well as the opportunities to learn more about pathophysiological mechanisms and targets that can also be applied to more common liver diseases. A lot has changed in technology, diagnostics and research approaches since 2006, when the authors of this editorial compiled for the Italian Association for the Study of the Liver (AISF) a first list of 75 rare liver diseases (24). It was useful to develop networks, a key component in the care of these patients. Nowadays however, patients with rare diseases need to be referred to centers specially designed to have the knowledge, the equipment and the technical skills. As in many examples of modern medicine, objective planning and adequate funding is paramount. For example, the National Institute of Health (NIH) has launched an Undiagnosed Disease Program, localized initially in the Bethesda Campus, before branching off to other sites in major US Universities. Similar programs, or integrated practice/research units are also advocated by the Value Based Medicine philosophy (25), which orients the health care delivery around the added value for the patient. Added value for a patient with a rare or undiagnosed liver disease is not touring health care centers for 5 years before reaching a diagnosis! Added value is being referred to an integrated practice/research unit specifically designed for these challenging diseases.

\section{Acknowledgments}

Funding: MS and LF would like to acknowledge the support of the Yale University Liver Center (P30DK034989), Clinical\&Traslational and Cellular\&Molecular cores. LF was also supported by Progetti di Ricerca di Dipartimento (PRIDDMM) 2017, University of Padua. MS acknowledges the support of the NIH grant 2R01_DK101528-05.

\section{Footnote}

Provenance and Peer Review: This article was commissioned by the editorial office, Translational Gastroenterology and Hepatology for the series "Recent Advances in Rare Liver Diseases". The article has undergone external peer review.

Conflicts of Interest: Both authors have completed the ICMJE uniform disclosure form (available at http://dx.doi.org/10.21037/ tgh-2020-05). The series "Recent Advances in Rare Liver Diseases" was commissioned by the editorial office without any 
funding or sponsorship. LF and MS served as the unpaid Guest Editors of the series. LF serves as an unpaid editorial board member of Translational Gastroenterology and Hepatology from Sep 2018 to Aug 2020. MS reports personal fees from Bayer, personal fees from ESIAI, personal fees from ENGITIX, outside the submitted work.

Ethical Statement: The authors are accountable for all aspects of the work in ensuring that questions related to the accuracy or integrity of any part of the work are appropriately investigated and resolved.

Open Access Statement: This is an Open Access article distributed in accordance with the Creative Commons AttributionNonCommercial-NoDerivs 4.0 International License (CC BY-NC-ND 4.0), which permits the non-commercial replication and distribution of the article with the strict proviso that no changes or edits are made and the original work is properly cited (including links to both the formal publication through the relevant DOI and the license). See: https://creativecommons.org/ licenses/by-nc-nd/4.0/.

\section{References}

1. Rare diseases. Public Health - European Commission. 2016 [cited 2020, 4th June 2020]. Available online: https://ec.europa.eu/ health/non_communicable_diseases/rare_diseases_en

2. Richter T, Nestler-Parr S, Babela R, et al. Rare Disease Terminology and Definitions-A Systematic Global Review: Report of the ISPOR Rare Disease Special Interest Group. Value Health 2015;18:906-14.

3. Allies in Rare Disease. Global Genes. [cited 2020, 4th June 2020]. Available online: https://globalgenes.org/

4. Orphanet. [cited 2020, 4th June 2020]. Available online: https://www.orpha.net/consor/cgi-bin/index.php?lng=EN

5. Tifft CJ, Adams DR. The National Institutes of Health undiagnosed diseases program. Curr Opin Pediatr 2014;26:626-33.

6. Moors EHM, Faber J. Orphan drugs: Unmet societal need for non-profitable privately supplied new products. Res Policy 2007;36:336-54.

7. Piperno A, Pelucchi S, Mariani R. Inherited iron overload disorders. Transl Gastroenterol Hepatol 2020;5:25.

8. Jones DEJ, Sturm E, Lohse AW. Access to care in rare liver diseases: New challenges and new opportunities. J Hepatol 2018;68:577-85.

9. Oliveira AG, Fiorotto R. Novel approaches to liver disease diagnosis and modeling. Transl Gastroenterol Hepatol 2020;6:19.

10. Fabris L, Fiorotto R, Spirli C, et al. Pathobiology of inherited biliary diseases: a roadmap to understand acquired liver diseases. Nat Rev Gastroenterol Hepatol 2019;16:497-511.

11. Moini M, To U, Schilsky ML. Recent advances in Wilson disease. Transl Gastroenterol Hepatol 2020;6:21.

12. Wang B. The acute hepatic porphyrias. Transl Gastroenterol Hepatol 2020;6:24.

13. Patel D, McAllister SL, Teckman JH. Alpha-1 antitrypsin deficiency liver disease. Transl Gastroenterol Hepatol 2020;6:23.

14. Gilbert MA, Loomes KM. Alagille syndrome and non-syndromic paucity of the intrahepatic bile ducts. Transl Gastroenterol Hepatol 2020;6:22.

15. Lasagni A, Cadamuro M, Morana G, et al. Fibrocystic liver disease: novel concepts and translational perspectives. Transl Gastroenterol Hepatol 2020;6:26.

16. Nastasio S, Matarazzo L, Sciveres M, Maggiore G. Giant cell hepatitis associated with autoimmune hemolytic anemia: an update. Transl Gastroenterol Hepatol 2020;6:25.

17. Weismüller TJ, Wedemeyer J, Kubicka S, et al. The challenges in primary sclerosing cholangitis--aetiopathogenesis, autoimmunity, management and malignancy. J Hepatol 2008;48 Suppl 1:S38-57.

18. Rabiee A, Silveira MG. Primary Sclerosing Cholangitis. Transl Gastroenterol Hepatol 2020;6:29.

19. Calvisi DF, Solinas A. Hepatoblastoma: current knowledge and promises from preclinical studies. Transl Gastroenterol Hepatol 2020;5:42.

20. Jain D, Torres R, Celli R, et al. Evolution of the liver biopsy and its future. Transl Gastroenterol Hepatol 2020;6:20.

21. Gao E, Hercun J, Heller T, Vilarinho S. Undiagnosed liver diseases. Transl Gastroenterol Hepatol 2020;6:28.

22. Finotti M, Auricchio P, Vitale A, Gringeri E, Cillo U. Liver transplantation for rare liver diseases and rare indications for liver transplant. Transl Gastroenterol Hepatol 2020;6:27. 
23. Trevisan M, Masi G, Palù G. Genome editing technologies to treat rare liver diseases. Transl Gastroenterol Hepatol $2020 ; 5: 23$.

24. Report Commissione Malattie Rare in Epatologia. AISF - ASSOCIAZIONE ITALIANA STUDIO DEL FEGATO. [cited 2020, 4th June 2020]. Available online: https://www.webaisf.org/wp-content/uploads/2019/01/commissione_malattie_rare_in_ epatologia.pdf

25. Strazzabosco M, Allen JI, Teisberg EO. Value-Based Medicine in Hepatology. Hepatology 2017;65:1749-55.

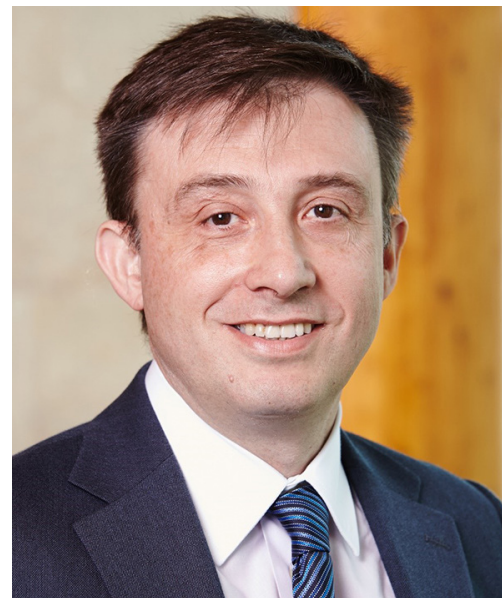

Luca Fabris

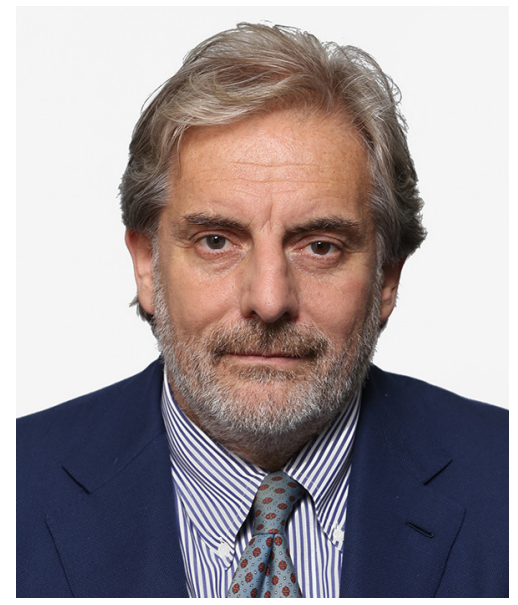

Mario Strazzabosco

\section{Luca Fabris ${ }^{1,2,3}$ \\ (Email: luca.fabris@unipd.it) \\ Mario Strazzabosco \\ (Email: mario.strazzabosco@yale.edu) \\ ${ }^{1}$ Department of Molecular Medicine, University of Padua, Padua, Italy; \\ ${ }^{2}$ General Medicine Division, University-Hospital, Padua, Italy; \\ ${ }^{3}$ Liver Center and Section of Digestive Diseases, Department of Internal Medicine, \\ Yale University School of Medicine, New Haven, CT, USA. \\ Received: 09 June 2020. Accepted: 27 July 2020; Published: 05 April 2021. \\ doi: $10.21037 / \mathrm{tgh}-2020-05$ \\ View this article at: http://dx.doi.org/10.21037/tgh-2020-05}

doi: $10.21037 / \operatorname{tgh}-2020-05$

Cite this article as: Fabris L, Strazzabosco M. Rare and undiagnosed liver diseases: challenges and opportunities. Transl Gastroenterol Hepatol 2021;6:18. 